NASA TN D-1042

TECHNICAL NOTE

D-1042

\title{
DENSITY IN A PLANETARY EXOSPHERE
}

\author{
Jackson Herring \\ and \\ Herbert L. Kyle
}

Goddard Space Flight Center

Greenbelt, Maryland

NATIONAL AERONAUTICS AND SPACE ADMINISTRATION 


\title{
DENSITY IN A PLANETARY EXOSPHERE
}

\author{
by \\ Jackson Herring \\ and \\ Herbert L. Kyle \\ Goddard Space Flight Center
}

\begin{abstract}
SUMMARY
A discussion of the Öpik-Singer theory of the density of a planetary exosphere is presented. Their density formula permits the calculation of the depth of the exosphere. Since the correctness of their derivation of the basic formula for the density distribution has been questioned, an alternate method based directly on Liouville's theorem is given. It is concluded that the Öpik-Singer formula seems valid for the ballistic component of the exosphere; but for a complete description of the planetary exosphere, the ionized and boundorbit components must also be included.
\end{abstract}



CONTENTS

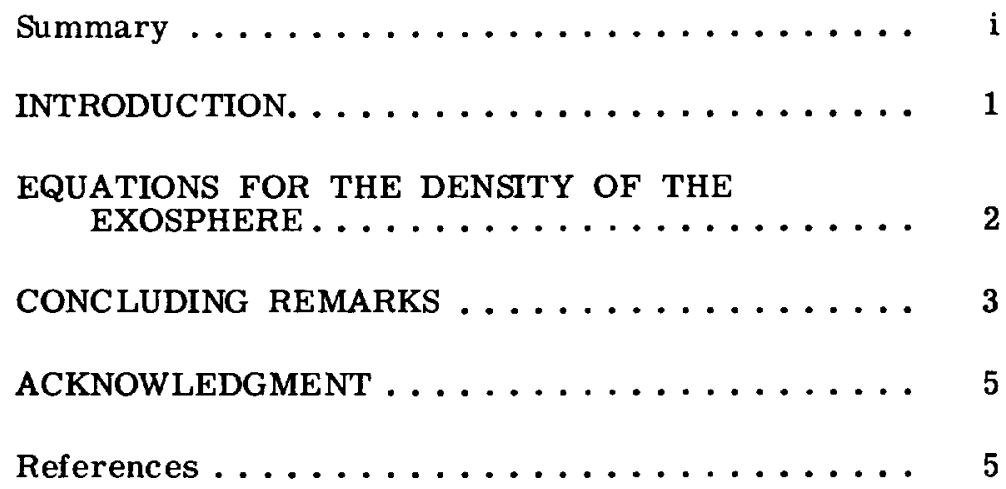




\title{
DENSITY IN A PLANETARY EXOSPHERE
}

\author{
by \\ Jackson Herring \\ and \\ Herbert L. Kyle \\ Goddard Space Flight Center
}

\section{INTRODUCTION}

Recently Öpik and Singer (Reference 1) have developed a theory which gives the ballistic component of the neutral density distribution in a planetary exosphere. Their theory assumes that above the base of the exosphere, collisions may be entirely neglected - at least as far as an approximate calculation of the neutral density profile is concerned. The particles at the base of the exosphere are assumed to be in a truncated Maxwellian distribution, with no incoming particles at greater than escape velocities. The absence of such incoming particles implies a sink, located at srine distance from the planet, which prevents the eventual build-up of a full Maxwellian distribution above the escape level; and this prevents an extension of the barometric law beyond the base of the exosphere. The bound-orbit and ionized components of the exosphere are omitted in the original OpikSinger development (however, see Reference 2). Other theories of the exosphere have been developed by Johnson and Fish (Reference 3) and by Chamberlain (Reference 4).

This paper will present several comments on the Öpik-Singer theory of the exosphere. First, since the correctness of their derivation of the basic formula for the density distribution (Equation 12 of Reference 1) has been questioned (Reference 5), it is worthwhile to present an alternate derivation of their formula for the density, based directly on Liouville's theorem. Second, a relatively simple analytic expression for the density distribution can be obtained which replaces the numerical integration required in the Öpik-Singer theory. The density formula permits a calculation of the depth of the exosphere, that is, the number of particles per unit area in a column extending from the base of the exosphere to infinity. This quantity is relevant to calculations of the escape of a planetary atmosphere. 


\section{EQUATIONS FOR THE DENSITY OF THE EXOSPHERE}

The formula for the density $\rho(\mathrm{r})$ can be derived directly from the one-particle form of Liouville's theorem, which states that the density of particles in phase space, $f(r, \vec{v})$ is constant along particle trajectories:

$$
f(\vec{r}, \vec{v})=f\left(\vec{R}, \vec{v}_{0}\right),
$$

where $\vec{v}$ is the velocity of a particle at position $\vec{r}$ and $\vec{v}_{0}$ is the velocity that the same particle had at the base of the exosphere, located on a sphere at $\vec{R}$. In the absence of collisions, $\vec{v}, \vec{r}$ and $\vec{v}_{0}, \vec{R}$ are related by the conservation of energy and of angular momentum:

$$
\begin{gathered}
\mathrm{v}=\sqrt{\mathrm{v}_{0}^{2}-\frac{2 \mathrm{NG}}{\mathrm{R}}(1-\mathrm{Y})}, \\
\mathrm{v} \sin \theta=\mathrm{v}_{0} \mathrm{Y} \sin \theta_{0}
\end{gathered}
$$

where $M$ is the mass of the planet, $G$ is the gravitational constant, and $Y$ is $R / r$. The angles $\theta$ and $\theta_{0}$ are the angles the trajectory makes with the radius vector passing through the center of the planet. These angles are defined with respect to the orbital plane. We shall assume in the following discussion that the density and temperature at the base of the exosphere are constants and therefore independent of th: angular co-ordinates of $\vec{R}$. The spacial density $\rho(r)$ is then

$$
\rho(r)=\int f(\vec{r}, \vec{v}) d \vec{v} .
$$

Equation 1 then allows us to write:

$$
\rho(\mathbf{r})=\int f\left(\vec{R}, \vec{v}_{0}\right) d \vec{v}
$$

In Equations 3 and 4, the range of integration $d \vec{v}$ extencs over all velocity space compatible with Equations $2 \mathrm{a}$ and $2 \mathrm{~b}$; that is, only over those orbits intersecting the spherical surfaces at $\vec{R}$ and $\vec{r}$. In order to evaluate the integral in Equation 4 we introduce the Jacobian, $\mathbf{J}\left(\mathbf{v}, \theta / \mathbf{v}_{0}, \theta_{0}\right)$, which transforms the integration over $d \vec{v}$ to one over $d \vec{v}_{0}$ :

$$
\rho(r)=\int v^{2} \sin \theta f\left(\vec{R}, \vec{v}_{0}\right) J\left(\frac{v_{1} t}{v_{0}, t_{0}^{-}}\right) d v_{0} d \theta_{0} .
$$


The Jacobian may be evaluated by using Equations $2 \mathrm{a}$ and $2 \mathrm{~b}$ :

$$
J\left(\frac{v, \theta}{v_{0}, \theta_{0}}\right)=\left(\frac{v_{0}}{v}\right)^{2} Y \frac{\cos \theta_{0}}{\sqrt{1-\left(\frac{v_{0} Y}{v}\right)^{2} \sin ^{2} \theta_{0}^{2}}}
$$

Again using Equations 2a and 2b, we eliminate $v$ and $\theta$ from Equation 5 and use Equation 6:

$$
\rho(\mathbf{r})=Y^{2} \int f\left(\vec{R}, \vec{v}_{0}\right) \frac{v_{0}{ }^{3} \cos \theta_{0} \sin \theta_{0} d v_{0} d \theta_{0}}{\sqrt{v_{0}^{2}\left(1-Y^{2} \sin ^{2} \theta_{0}\right)-\frac{2 M G}{R}(1-Y)}} .
$$

Öpik and Singer's Equation 12 may be obtained from Equation 7 if we replace $f\left(\vec{R}, \vec{v}_{0}\right)$ by a truncated Maxwellian distribution which omits incoming particles with velocities greater than escape velocity.

The integration in Equation 7 can be performed to give $\rho(r)$ in terms of known functions:

$$
\begin{aligned}
\rho(\mathrm{r})=\rho_{0}(\mathrm{R})[ & \mathrm{e}^{-\alpha(1-\mathrm{Y})}\left(1-\frac{1}{2} \operatorname{erf} \sqrt{\alpha \mathrm{Y}}\right) \\
& \left.-\sqrt{1-\mathrm{Y}^{2}} \mathrm{e}^{-\frac{\alpha}{1+Y}}\left(1-\frac{1}{2} \operatorname{erf} \sqrt{\frac{\alpha Y}{1+\mathrm{Y}}}\right)-\sqrt{\frac{\alpha Y}{\pi}}(1-\sqrt{1-\mathrm{Y}}) \mathrm{e}^{-\alpha}\right],
\end{aligned}
$$

where

$$
\begin{aligned}
a & =\mathrm{R} / \mathrm{H}, \\
\mathrm{H} & =\text { scale height, } \\
\rho_{0}(\mathrm{R}) & =\text { density at the critical level, and } \\
\text { erf } \mathrm{x} & =\frac{2}{\sqrt{\pi}} \int_{\mathrm{x}}^{\infty} \mathrm{e}^{-\mathrm{Y}^{2}} \mathrm{dY} .
\end{aligned}
$$

\section{CONCLUDING REMARKS}

When considering the above distribution, we first notice that as $a \rightarrow \infty$, the barometric law for the density variation is obtained. Secondly, we observe that $\rho(R)$ is not equal to $\rho_{0}(R)$, the density just below the base of the exosphere. The reason for this is that we have 
omitted all incoming particles with velocities greater than escape velocity. This discontinuity results from arbitrarily neglecting all collisions abcive the escape level, and a more realistic theory would replace the sharp boundary of he escape level by a diffuse zone above which collisions gradually become less likely.

The density profile, Equation 8 , is characterized by a parameter $a=\mathrm{R} / \mathrm{H}$, but not in too transparent a way. This parameter determines the extent of the exosphere, and in order to gain some insight into this matter, we have calculated the quantity $d$ :

$$
\mathbf{d}=\int_{\mathbf{R}}^{\infty} \rho(\mathbf{r}) \mathrm{d} \mathbf{r} .
$$

In the conventional theory of escape of a planetary atmosphere, $d$ is taken to be equal to $\rho_{0} \mathrm{H}$, with $\mathrm{H}$ being the scale height at the base of the exosphere. In Figure 1, the ratio $\mathrm{d} / \rho_{0} \mathrm{H}$ is plotted as a function of $a$. For orientation, we may note that for the earth's exosphere $a=4.5$ for atomic hydrogen and $a \approx 72$ for atomic oxygen, provided we take a temperature of $1500^{\circ} \mathrm{K}$ at the base of the exosphere. In the limit as a approaches zero, $\mathbf{H}$ approaches infinity, $\mathbf{d}$ becomes independent of $a$, and $\rho$ jecomes proportional to $1 / \mathrm{r}^{2}$. For large values of $\alpha, \mathrm{d}$ approaches $\rho_{0} \mathrm{H}$; in other words, there is one scale height of atmosphere above the escape level. This result has been used by various investigators (Reference 6) in their studies of the escape of atmospheres, but their justification for its

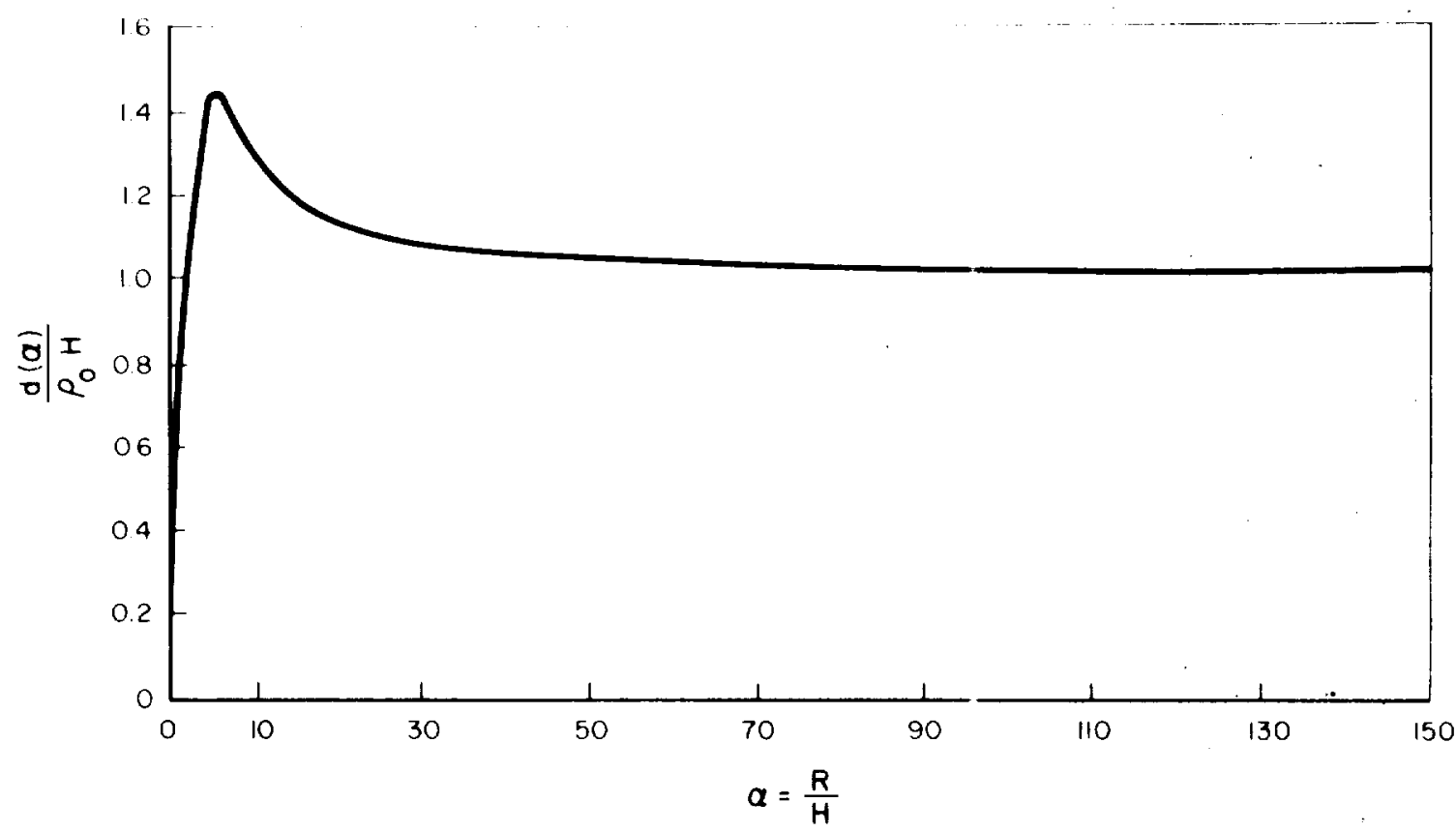

Figure 1 - Variation of the ratio $\mathrm{d} / \rho_{0} \mathrm{H}$ with the parameter $\alpha$. The integrated neutral exosphere density is given by $d$ in Equation 8 . In the conventional theory of the escape of a planetary atmosphere, $\mathrm{d}$ is set equal to $\rho_{0} \mathrm{H}$. 
use applies to stellar rather than to planetary atmospheres. The Öpik-Singer theory seems to provide a valid justification for its use in the case of the ballistic component of the exosphere; however, it should be stressed that the ionized and bound-orbit components must be included for a complete description of a planetary exosphere. For example, Singer (Reference 7) has suggested that in the earth's exosphere the dominant components above 1800 kilometers may be $0^{+}$and $\mathrm{H}^{+}$. Öpik and Singer have recently published a supplemental discussion of the bound orbit component. Their findings indicate that this component is unimportant in the earth's exosphere, but may be important in the exospheres of the major planets (Reference 2).

\section{ACKNOWLEDGMENT}

The authors wish to thank R. Roth of the Goddard Space Flight Center for the numerical integration of Equation 9 on the IBM 7090 computer.

\section{REFERENCES}

1. Öpik, E.J., and Singer, S.F., "Distribution of Density in a Planetary Exposphere," Physics of Fluids 2:653-655, November-December 1959

2. Öpik, E.J., and Singer, S.F., "Distribution of Density in a Planetary Exosphere. II," Physics of Fluids 4(2):221-233, February 1961

3. Johnson, F.S., and Fish, R.A., "The Telluric Hydrogen Corona," Astrophys. J. 131:502515, March 1960

4. Chamberlain, J.W., "Interplanetary Gas. II Expansion of a Model Solar Corona," Astrophys. J. 131:47-56, January 1960

5. Brandt, J.C., and Chamberlain, J.W., "Density of Neutral Gas in a Planetary Exosphere," Physics of Fluids 3:485-486, May-June 1960

6. Mitra, S.K., "The Upper Atmosphere," Calcutta: The Asiatic Society, 1952

7. Singer, S.F., "Structure of the Earth's Exosphere," J. Geophys. Res. 65:2577-2580, September 1960 


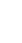

